\title{
Randomised controlled trial of sub-Tenon's block versus topical anaesthesia for cataract surgery: a comparison of patient satisfaction
}

\author{
H Rüschen, D Celaschi, C Bunce, C Carr
}

Br J Ophthalmol 2005;89:291-293. doi: 10.1136/bjo.2004.052167

Background/aim: Sub-Tenon's block (STB) or topical anaesthesia alone (TOP) are popular techniques employed during cataract surgery. TOP is often preferred by healthcare providers because of financial or staffing reasons, despite existing evidence that pain during surgery is better controlled with STB. Pain is not the only consideration that determines patient preference for the anaesthesia technique. The authors decided to investigate the issue of patient satisfaction using the recently developed lowa Satisfaction with Anesthesia Scale (ISAS)

Method: In a randomised controlled pilot trial, 28 patients were enrolled to receive either STB with $3 \mathrm{ml}$ of $2 \%$ lidocaine and hyaluronidase, or TOP with proxymetacaine $0.5 \%$ and amethocaine 1\% (Tetracaine) eye drops. Postoperatively patients rated their satisfaction with anaesthesia care by filling in the self administered written questionnaire, the ISAS. Results: One patient in the TOP group dropped out of the study because of intolerable pain. Analysis of the questionnaire results with a two sample Wilcoxon rank sum test showed a significant difference in patient satisfaction $(p<0.0085)$. The median satisfaction score was higher in the STB group 2.77 (interquartile range IQR 2.45 to 3), than in the TOP group 2.04 (IQR 1.54 to 2.5).

Conclusion: In the setting of day case cataract surgery, patients report significantly higher satisfaction scores with STB than with TOP alone.

\footnotetext{
C
} ataract surgery is the most frequent elective operation worldwide. In the United Kingdom 200000 cataract operations are carried out each year ( 1.5 million in the United States). A recent government initiative to reduce waiting time has led to the introduction of diagnostic and treatment centres (DTC) to provide streamlined care and high turnover surgery. But there is a substantial national and international variation in anaesthesia management strategies for cataract operations. Concerns about the safety of sharp needle blocks (peribulbar or retrobulbar) have led to increased use of blunt needle sub-Tenon's block (STB) and topical anaesthesia (TOP). A recent systematic review of local anaesthesia techniques for cataract removal found evidence that STB results in less pain during cataract surgery than TOP. ${ }^{1}$ There have been very few studies of sufficient quality that compare STB with TOP in this setting. ${ }^{23}$ These studies usually assess the pain experienced during surgery with a visual analogue scale (VAS). Patient satisfaction is determined by a multitude of factors, pain is only one of them.

The choice of using either STB or TOP for high numbers of cataract operations has important resource and staffing implications. When financial imperatives prompt changes in health care, we are obliged to do more with less, but the profession and the public will expect maintenance of quality. We decided to investigate the issue of patient satisfaction further, using a recently developed scoring system, the Iowa Satisfaction with Anesthesia Scale (ISAS). ${ }^{4}$

\section{METHODS}

In an attempt to determine which considerations are particularly important for older patients when they undergo cataract surgery, consumer input was sought from lobby groups and individuals. Their views were incorporated into the design of this pilot study as much as possible. A power estimate was not possible because there were no existing publications on this topic. After obtaining ethics committee approval, we conducted a randomised controlled pilot trial in the St Ann's cataract diagnostic and treatment centre (DTC), the satisfaction with local anaesthesia for cataract surgery trial (SLACS). During their preoperative assessment by DTC nurses, 28 consecutive patients were enrolled in the study according to the inclusion and exclusion criteria. Inclusion criteria were patient suitable for cataract surgery in the setting of a DTC according to locally established guidelines, first eye operation. The exclusion criteria were patient cannot understand the information about the trial for any reason, patient requested sedation for the operation, patient had significant other ocular morbidity apart from cataract. Patients were given an information leaflet describing the study at least 1 week ahead of surgery. Written informed consent was obtained on the day of surgery. Computer generated permuted block randomisation was obtained from the department of biostatistics via telephone. Both patients and surgeons were formally masked regarding the method of anaesthesia, but no sham injections were given in the TOP group.

Dropout criteria were if the patient voices that they want to drop out of the study, if the patient expresses uncontrolled pain, or if the patient becomes restless. It was also decided that patients who drop out should receive a top up, or injection of local anaesthetic block and midazolam sedation to continue the operation if possible. All local anaesthesia was administered by anaesthetists or surgeons, who were not otherwise involved in the care of that patient or analysis of satisfaction scores. The surgeons or anaesthetists were asked to give a standard STB with $3-4 \mathrm{ml}$ of lidocaine $2 \%$. The local anaesthetic contained 30 units per $\mathrm{ml}$ of hyaluronidase. The block was injected in the inferomedial quadrant of the eye, using a 19 gauge sub-Tenon's cannula (Visitech Sarasota, FL, USA). TOP consisted of two drops of proxymetacaine $0.5 \%$ followed by four drops of amethocaine $1 \%$ (Tetracaine). None

Abbreviations: DTC, diagnostic and treatment centre; ISAS, lowa Satisfaction with Anesthesia Scale; STB, sub-Tenon's block; TOP, topical anaesthesia alone 
of the patients received any other analgesia or sedation. Intravenous cannulae were not inserted. All surgical procedures were phacoemulsification and intraocular lens implantation. Intracameral local anaesthesia was not used. Apart from demographic data the following variables were recorded: medical or surgical disease, waiting time from first symptoms of cataract, access to hospital transport, duration of surgery, complications of anaesthesia or surgery, experience of anaesthetist and surgeon. No important differences between the groups were identifiable that would have influenced satisfaction scoring. The instrument for measuring patient satisfaction is the Iowa Satisfaction with Anesthesia Scale (ISAS), a self administered written questionnaire. This tool has been developed and tested to be an internally consistent, reliable, and valid measure of patient satisfaction. It was designed to measure the patients' satisfaction specifically with their anaesthesia care, or what patients believe to be part of that. Patients respond to 11 statements (for example, I felt pain) by placing a mark along a six choice vertical response column (for example, Disagree moderately) below each statement (boxes 1 and 2).

A totally satisfied patient would score +3 , a totally dissatisfied patient would score -3 . The mean of their responses to the 11 statements gives a single number between -3 and +3 , which is a quantitative measure of a patient's satisfaction with their monitored anaesthesia care (MAC). Patients were handed the questionnaire as soon as they recovered after the operation. The time to fill in the form was not limited. None of the investigators or operators was in contact with the patient during this time.

\section{RESULTS}

Randomisation resulted in 14 patients being allocated to each group, STB or TOP. Both groups were comparable regarding age, sex, waiting time, ASA status, access to hospital transport, surgical time (table 1 ).

\section{Box 1 ISAS questions}

(1) I was too cold or hot

(2) I would want to have the same anaesthetic again

(3) I itched

(4) I felt relaxed

(5) I felt pain

(6) I felt safe

(7) I threw up or felt like throwing up

(8) I was satisfied with my anaesthetic care

(9) I felt pain during surgery

(10) I felt good

(11) I hurt

\section{Box 2 ISAS responses}

- Agree very much

- Agree moderately

- Agree slightly

- Disagree slightly

- Disagree moderately

- Disagree very much

\begin{tabular}{|lccl|}
\hline Table 1 & Patient characteristics & & \\
\hline & STB & TOP & Significance \\
\hline Age & 70.4 & 72.6 & \\
Access to hospital transport & 2 & 3 & \\
Females & 5 & 12 & NS \\
Waiting more than 3 months & 12 & 9 & NS \\
ASA 1 & 7 & 7 & \\
ASA 2 & 7 & 6 & \\
ASA 3 & 0 & 1 & \\
Surgical time (minutes) & 23.5 & 22.5 & \\
\hline
\end{tabular}

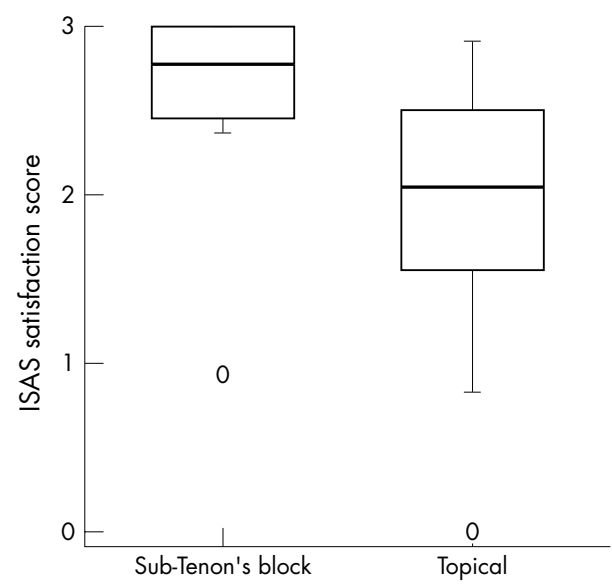

Figure 1 Box and whisker plot of satisfaction score with sub-Tenon's block or topical anaesthesia.

One patient dropped out after randomisation (TOP). Ten minutes into the operation the patient expressed severe pain and was moving too much for the surgeon to continue operating. He was given $1 \mathrm{mg}$ midazolam and a peribulbar block to complete the surgery. Two of the 28 questionnaires contained unanswered questions, both in the TOP group. Both questionnaires were not included in the analysis. Two sample Wilcoxon rank sum test was performed on the questionnaire results. The analysis shows evidence of a significant difference in patient satisfaction, with higher scores being obtained for the STB group than the TOP group $(\mathrm{p}<0.0085)$. The median satisfaction score in the TOP group was 2.04 (interquartile range 1.54 to 2.50 ), as opposed to 2.77 (IQR 2.45 to 3 ) in the STB group (fig 1).

\section{DISCUSSION}

This is the first study to investigate levels of patient satisfaction after cataract surgery using a validated reliable and internally consistent assessment tool. STB is known to produce more profound anaesthesia and analgesia than TOP, as shown by previous studies. Despite that, the number of operations carried out under topical anaesthesia is rising. This might be secondary to economical considerations, or perhaps the result of the perception that patients do not mind pain as long as it is short lived. Patient satisfaction is a direct marker for quality of care. We believe the results of this study provide evidence to guide policies in favour of STB over simply using TOP.

\section{CONCLUSION}

In the setting of cataract surgery in a day treatment centre, sub-Tenon's block produces significantly higher satisfaction scores than topical anaesthesia alone. 


\section{ACKNOWLEDGEMENTS}

We thank all the staff of St Ann's DTC: doctors, nurses, ODAs, and clerical staff for their help with the conduct of the study. Thanks to Wen Xing for providing randomisation, and to Professor F Dexter for allowing us to use the ISAS.

\section{Authors' affiliations}

H Rüschen, D Celaschi, C Bunce, C Carr, Moorfields Eye Hospital, St Ann's Diagnostic and Treatment Centre, City Road, London ECIV 2PD, UK

Competing interests: none declared.

The results of this trial were presented at the OAS meeting in Chicago, October 2003.
Correspondence to: Heinrich Rüschen, Moorfields Eye Hospital, 162 City Road, London ECIV 2PD, UK; anaesthetic.research@moorfields.nhs.uk

Accepted for publication 22 July 2004

\section{REFERENCES}

1 Friedman DS, Bass EB, Lubomski LH. Synthesis of the literature on the effectiveness of regional anesthesia for cataract surgery. Ophthalmology 2001;108:519-29.

2 Manners TD, Burton RL. Randomised trial of topical versus sub-Tenon's local anaesthesia for small-incision cataract surgery [see comments]. Eye 1996; 10(Pt 3):367-70.

3 Zafirakis P, Voudouri A, Rowe S, et al. Topical versus sub-Tenon's anesthesia without sedation in cataract surgery. J Cataract Refract Surg 2001;27:873-9.

4 Dexter F, Aker J, Wright WA. Development of a measure of patient satisfaction with monitored anesthesia care: the lowa Satisfaction with Anesthesia Scale. Anesthesiology 1997;87:865-73.

\section{$\mathrm{ECHO}$}

\section{Clot busting in situ may be best for treating a blocked central retinal artery}

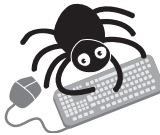

Please visit the British Journal of

Ophthalmology website [www. bjophthalmol. com] for a link to the full text of this article. ntra-arterial thrombolysis (IAT) may be a better way of restoring vision in patients with sudden blockage of the central retinal artery in future, if promising results from an initial study are confirmed by randomised control trial.

Patients having IAT with urokinase in the retrospective case-control study had a better chance of improved visual acuity, with more attaining a final visual acuity of $>0.6$ than those having conventional treatment $(22 \% v 0 \%)$. Both patient groups were well matched. Younger age was the only significant attribute linked to improved vision, but the researchers are reluctant to use this to select for treatment as improvement did occur in some older some patients and because the condition has such a poor prognosis generally. Six patients had adverse events: cerebrovascular ischaemia, transient ischaemic attacks, and a stroke, promptly located and treated with urokinase.

The study compared 37 patients having IAT with urokinase to disperse a clot in a central retinal artery within six hours after the event with 19 patients who received conventional treatment-aspirin or heparin with anterior chamber paracentesis or acetazolamide, or both-because of unavailability of IAT or unfavourable circumstances. Eyesight was assessed by standard measurement within 48 hours afterwards and 2-8 weeks later.

Several case series and a recent meta-analysis have indicated that IAT may be a promising treatment, but because of haemorrhagic or ischaemic complications the researchers caution that the procedure should be performed by a neuroradiologist familiar with doing it in vessels within the brain too.

A Arnold M, et al. Journal of Neurology, Neurosurgery, and Psychiatry 2005;76:196-199. 